\title{
Probing Size-Dependent Electrokinetics of Hematite Aggregates
}

\author{
Karolina Kędra-Królik ${ }^{\mathrm{a}}$, Kevin M. Rosso ${ }^{\mathrm{b}}$, Piotr Zarzycki ${ }^{\mathrm{a}, *}$ \\ ${ }^{a}$ Institute of Physical Chemistry, Polish Academy of Sciences, Kasprzaka 44/52, 01-224 Warsaw, Poland \\ ${ }^{b}$ Pacific Northwest National Laboratory, Richland, WA
}

\begin{abstract}
Aqueous particle suspensions of many kinds are stabilized by the electrostatic potential developed at their surfaces from reaction with water and ions. An important and less well understood aspect of this stabilization is the dependence of the electrostatic surface potential on particle size. Surface electrostatics are typically probed by measuring particle electrophoretic mobilities and quantified in the electrokinetic potential $(\zeta)$, using commercially available Zeta Potential Analyzers (ZPA). Even though ZPAs provide frequency-spectra (histograms) of electrophoretic mobility and hydrodynamic diameter, typically only the maximal-intensity values are reported, despite the information in the remainder of the spectra. Here we propose a mapping procedure that inter-correlates these histograms to extract additional insight, in this case to probe particle size-dependent electrokinetics. Our method is illustrated for a suspension of prototypical iron (III) oxide (hematite, $\alpha-\mathrm{Fe}_{2} \mathrm{O}_{3}$ ). We found that the electrophoretic mobility and $\zeta$-potential are a linear function of the aggregate size. By analyzing the distribution of surface site types as a function of aggregate size we show that site coordination increases with increasing aggregate diameter. This observation explains why the acidity of the iron oxide particles decreases with increasing particle size.
\end{abstract}

Keywords: zeta potential, electrokinetics, hematite, $\alpha-\mathrm{Fe}_{2} \mathrm{O}_{3}$, iron oxide/electrolyte interface, electrical double layer, electrophoresis, Central Limit Theorem, electrophoretic mobility, particle size

\section{Introduction}

Most particles immersed in an aqueous solution develop a surface charge that stabilizes their suspension [1]. The charged surface attracts electrolyte ions resulting in a spatial charge distribution known as the electrical double layer (EDL) $[1,2]$.

If charged particles are subjected to an external electric field, they move in the field together with a part of the surrounding ionic atmosphere (i.e., rigid part of EDL) [1]. The electrostatic potential developed at the boundary between this rigid part and the remaining EDL structure is referred to as the electrokinetic potential ( $\zeta$-potential) [1]. The $\zeta$-potential is one of the most important EDL-descriptors, essential in assessing suspension stabilities, bacteria/viruses adhesion, and biomolecules surface electrostatics (e.g., proteins, liposomes, micelles) $[3,4,5,6,7,8]$. The $\zeta$-potential is calculated from the electrophoretic mobility $\left(u_{e}\right)$, which is a readily accessible property in a variety of experimental techniques based on the electrokinetic phenomena, however it is the most frequently obtained using Zeta Potential Analyzers (ZPA).

Commercially available ZPAs can record the electrophoretic mobility of suspended charged particles and their hydrodynamic diameter by exploiting the phenomenon of dynamic light scattering (DLS) $[9,10,11,12,13]$. In typical settings, ZPA provides frequency spectra (histograms) of the electrophoretic mobility or corresponding $\zeta$ potential and particle hydrodynamic diameter $\left(d_{\text {hydro }}\right)$. Unfortunately, only the average or maximal-intensity values are usually reported, thus a large amount of data associated with the shapes of these spectra are typically ignored despite their huge potential value.

${ }^{*}$ Corresponding author

Email address: zarzycki .piotrek@gmail. com (Piotr Zarzycki) 
In this report we propose a method to more fully utilize available data by mapping $u_{e}$ (or $\zeta$ ) and $d_{\text {hydro }}$ distributions, which we will show can allow one to gain insight into the size-dependency of particle surface electrostatics. We illustrated our method for the prototypical iron (III) oxide hematite $\left(\alpha-\mathrm{Fe}_{2} \mathrm{O}_{3}\right)$. Hematite is a naturally occurring semi-conductor, and one of the most stable iron (III) oxide minerals among a wide range of possible iron oxide phases. On the basis of the Kelvin equation it is generally accepted that the reactivity of hematite particles will increase with decreasing particle size, however at the nanoscale particle stability is not easily predictable and much is left to be understood [14]. Although our procedure is illustrated for metal oxide particles, we believe that it can be applied to other suspensions.

\section{Materials and protocols}

Hematite $\left(\alpha-\mathrm{Fe}_{2} \mathrm{O}_{3}\right)$ nanoparticles (20-40 nm in diameter) were purchased from SkySpring Nanomaterials Inc. (99\% $\alpha-\mathrm{Fe}_{2} \mathrm{O}_{3}$ ). The $\zeta$-potential and hydrodynamic diameter were measured by a commercially available ZPA (NanoBrook 90Plus Zeta Potential Analyzer, Brookhaven Instruments Corporation, US). The titrations were carried out under argon atmosphere. All solutions were prepared using the distilled and deionized water and analytical grade electrolytes $\left(\mathrm{KNO}_{3}, \mathrm{KOH}, \mathrm{HNO}_{3}\right)$. Particle suspensions were prepared 12 hours before titrations in $0.001 \mathrm{~mol} / \mathrm{dm}^{3}$ $\mathrm{KNO}_{3}$ electrolyte and kept at $\mathrm{pH}=3$.

\section{Methodology}

Experiment. Quasi-elastic dynamic light scattering (DLS) is the phenomenon behind the diameter and electrophoretic mobility measurements presented in this report. The DLS-apparatus measures the intensity autocorrelation function of light scattered by the suspended particles $(c(t))$. The particles are subjected to Brownian and electrophoretic (in case of the $\zeta$-measurement) forces, resulting in a fluctuation of scattered light intensity (Doppler effect) $[15,16]$.

Analysis. The normalized $c(t)$ function is related to a particle diffusion coefficient via the dynamic structure factor providing that the magnitude of the scattered electric field (or intensity) obeys Gaussian statistics (Siegert relationship) [16]. By knowing the diffusion coefficient $(D)$ one can calculate the hydrodynamic particle diameter, for instance by using the Stokes-Einstein relationship: $d_{\text {hydro }}=k_{B} T /(3 \pi \eta D)$ (where $k_{B}$ is the Boltzmann constant, $\eta$ is a medium viscosity, and $T$ is temperature). In order to convert electrophoretic mobility to $\zeta$-potential, an appropriate electrokinetic theory has to be used for a given double-layer thickness and particle radius [17]. The thickness of the EDL is defined by the Debye length $\kappa^{-1}$ as:

$$
\kappa^{-1}=\sqrt{\frac{\epsilon \epsilon_{0} k_{B} T}{N_{A} \sum_{i}^{i o n s} e^{2} z_{i}^{2} c_{i}}}
$$

where $\epsilon$ is the dielectric constant for solution, $\epsilon_{0}$ is the vacuum permittivity, $k_{B}$ is the Boltzmann constant, $T$ is temperature, $z_{i}$ and $c_{i}$ are the charge number and molar concentration of ion $i$. In the case presented here (i.e., 0.001 $\left.\mathrm{mol} / \mathrm{dm}^{3} \mathrm{KNO}_{3}, \mathrm{~T}=25^{\circ} \mathrm{C}, \epsilon=78.54\right)$ the EDL thickness $\left(\kappa^{-1}\right)$ is equal to $9.62 \mathrm{~nm}$.

In the case of a thin double layer $\left(\kappa^{-1} \ll a\right.$, where $a=d_{\text {hydro }} / 2$ is a particle radius), the $\zeta$-potential is given by the Smoluchowski relation (or Helmholtz-Smoluchowski) [17]:

$$
\zeta=\frac{u_{e} \eta}{\epsilon \epsilon_{0}}
$$

In the case of a thick double layer $\left(\kappa^{-1} \gg a\right)$, the $\zeta$-potential is related to the electrophoretic mobility via Hückel equation (or Hückel-Onsager) [17]:

$$
\zeta=\frac{3}{2} \frac{u_{e} \eta}{\epsilon \epsilon_{0}}
$$

To convert $u_{e}$ to $\zeta$ for systems in the $\kappa a$ range between thick and thin EDL extremes one can use Henry's formula [17]:

$$
\zeta=\frac{3}{2} \frac{u_{e} \eta}{\epsilon \epsilon_{0} f_{1}(\kappa a)}
$$


where $f_{1}(\kappa a)$ is the Henry's function that varies smoothly between 1 ( $\kappa a \ll 1$, Hückel) to 1.5 ( $\kappa a \gg 1$, Smoluchowski). The Henry's function can be approximated by the following Ohshima's relation [18, 19]:

$$
f_{1}(\kappa a)=1+\frac{1}{2}\left[1+\left(\frac{2.5}{\kappa a(1+2 \exp (-\kappa a))}\right)\right]^{3}
$$
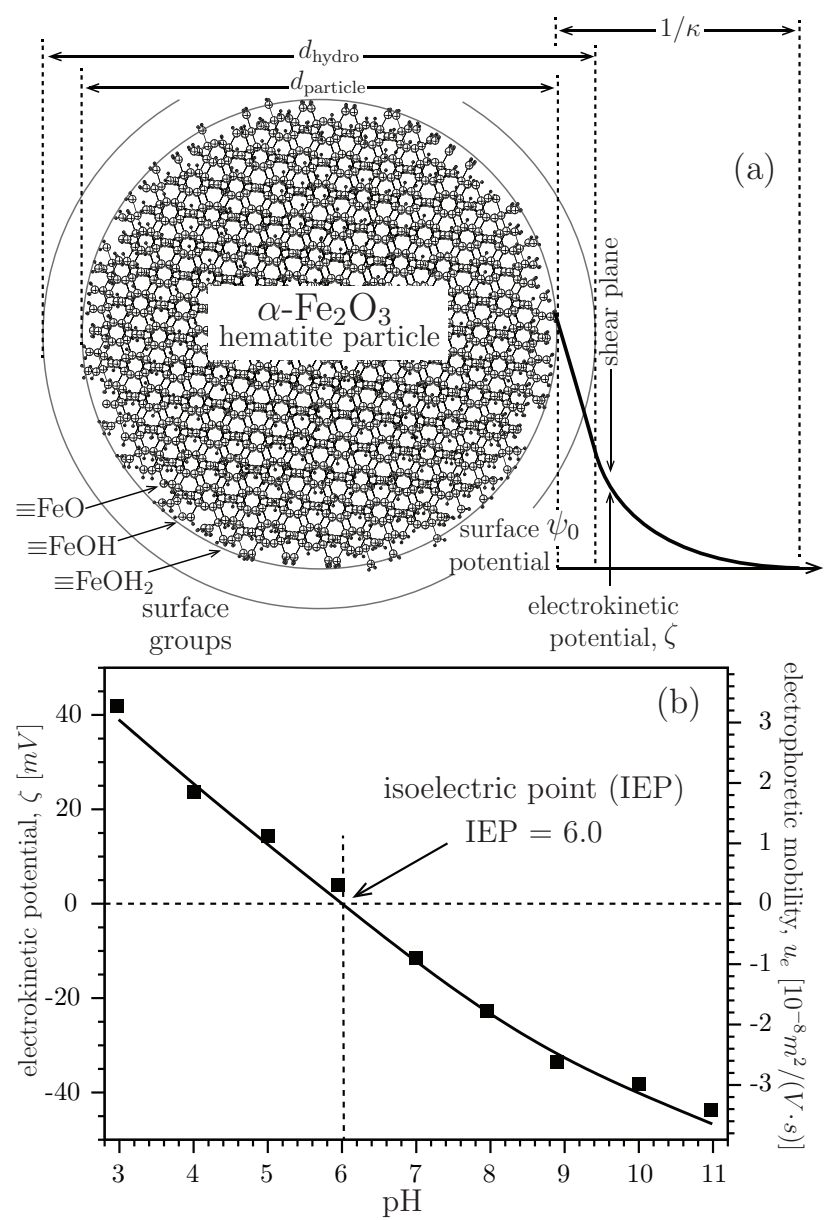

Figure 1: Schematic model of the electrical double layer (EDL) developed at the spherical $\alpha-\mathrm{Fe}_{2} \mathrm{O}_{3}$ particle/electrolyte interface (a): electrokinetic potential $(\zeta)$ forms at a boundary between the rigid and mobile parts of EDL (i.e., at the shear plane). In panel (b) we show the electrokinetic curve ( $\zeta$-potential and electrophoretic mobility, $\left.u_{e}\right)$ for our hematite particles $\left(\langle\zeta\rangle=\mathrm{f}(\mathrm{pH}),\left\langle u_{e}\right\rangle=\mathrm{f}(\mathrm{pH})\right.$, isoelectric point is the $\mathrm{pH}$ value at which $\langle\zeta\rangle=\left\langle u_{e}\right\rangle$ $=0)$.

Mapping probability distributions. In this report we propose to fully utilize the data collected by a commercial ZPA to gain an insight into the particle-size-dependent electrostatics. We propose to map the particle size $\left(d_{\text {hydro }}\right)$ onto electrophoretic mobility $\left(u_{e}\right)$ by exploiting the reversibility[20] of their normalized probability distributions $(P)$. Provided that proportionality relationship between $u_{e}$ and $d_{\text {hydro }}$ is known, then by setting $P^{-1}\left(u_{e}\right)=P^{-1}(d)$ one can get $u_{e}=f\left(d_{\text {hydro }}\right)$ (where $P^{-1}$ is an inverse of $P$ ).

On the one hand, this mapping procedure does not require any specific functional form of $P$, provided that a reversible (or piecewise reversible) representation can be established. Here however, we approximated the experimental histograms by a Gaussian function for the sake of computational simplicity.

On the other hand, one may notice that Gaussian statistics are inherent to many aspects of the DLS measurements. For instance, the Doppler broadening of scattered light frequencies (phenomena behind dynamic scattering) obeys the Gaussian distribution with a width of peak at half-height related to the particle diffusion coefficient [2]. The 
intensity autocorrelation function - the quantity directly measured in DLS - is also related to the structure factor only if the magnitude of scattered electric field/light intensity is described by a Gaussian distribution [16]. What is more, the positional correlation function, which is related to the structure factor via the Fourier transform, is also given by Gaussian statistics providing particles are subjected only to random (Brownian) motion [21]. It is of course a manifestation of how important Gaussian statistics is in physicochemical measurements (cf. Central Limit Theorem, CLT) $[22,23]$. In summary, one could expect that the size and $u_{e}$ histograms provided by commercially available ZPAs should in principle be Gaussian. This is consistent with an assumption that the DLS measurement is carried out in a linear-response regime as well as the fact that light received by a detector is a superposition of independently scattered photons $[15,23]$.

In summary, our mapping procedure consists of the following steps (see Supporting Information for details):

1. calculate normalized distribution functions $P\left(u_{e}\right), P\left(d_{\text {hydro }}\right)$ from the experimental histograms of $u_{e}$ and $d_{\text {hydro }}$

2. calculate inverse functions: $u_{e}=f^{-1}(P)$ and $d_{\mathrm{d}_{\mathrm{h}}}=f^{-1}(P)$

3. if $|\zeta|$ increases with an increasing particle size then map $P\left(d_{\text {hydro }}\right) \rightarrow P\left(-u_{e}\right)$ for $\mathrm{pH}>\operatorname{IEP}$ and $P\left(d_{\text {hydro }}\right) \rightarrow P\left(u_{e}\right)$ for $\mathrm{pH}<\mathrm{IEP}$; if $\mid \zeta$ decreases with increasing particle size then reverse the mapping.
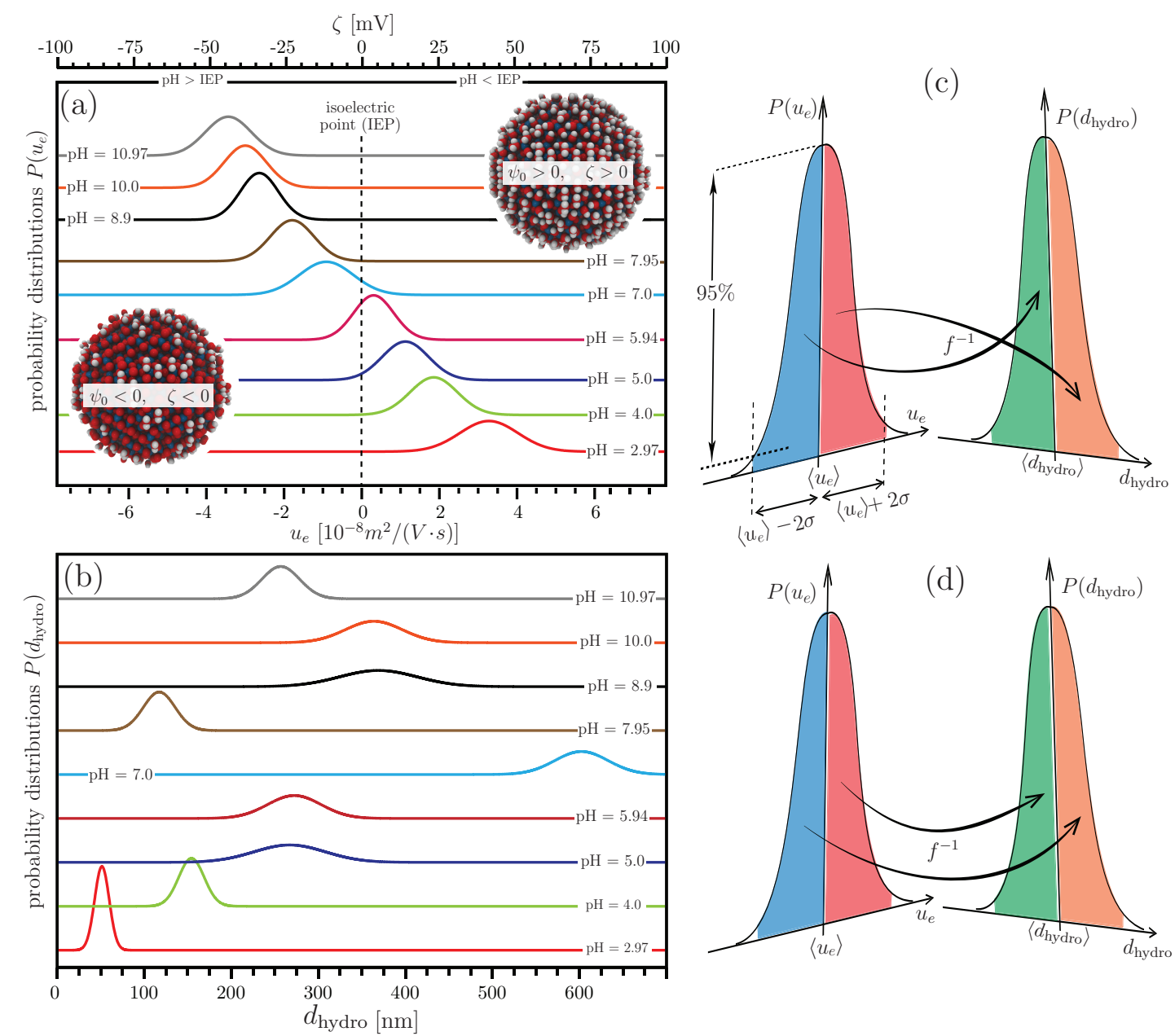

Figure 2: Gaussian-approximated histograms of electrophoretic mobility, $u_{e}$ (a) and hydrodynamic diameter (b). A mapping procedure $\left(P\left(u_{e}\right) \rightarrow\right.$ $\left.P\left(d_{\text {hydro }}\right)\right)$ is illustrated in the panels: (c) reverse mapping $P\left(d_{\text {hydro }}\right) \rightarrow P\left(-u_{e}\right)$ and (d) direct mapping $P\left(d_{\text {hydro }}\right) \rightarrow P\left(u_{e}\right)$. The distribution domains (i.e., range of $u_{e}$ and $d_{\text {hydro }}$ values) are divided into two reversible subdomains (red/blue for $u_{e}$ and green/orange for $d_{\text {hydro }}$ ) taking into account only $95 \%$ of the recorded values (empirical three-sigma rule, see Supporting Information for details). One can also map the hydrodynamic diameter into $\zeta$-potential providing that correct electrokinetic theory is applied to convert $u_{e}$ into $\zeta$ (see discussion in ref. [17]). 


\section{Results and discussion}

In Fig. 1a we show a schematic representation of the EDL formed at nanoparticle hematite/electrolyte interfaces. The surface electrostatics of oxide minerals is primarily governed by the solution $\mathrm{pH}$. The surface charge of oxide particles is formed due to proton uptake and release by surface-exposed hydroxyl groups, therefore the EDL descriptors (here $\zeta$-potential) are typically reported as a function of $\mathrm{pH}[2,24]$. Electrophoretic mobility and $\zeta$-potential have positive values in acidic and negative in alkaline $\mathrm{pH}$ region. In particular, the $\mathrm{pH}$ value at which $u_{e}=\zeta=0$ is a unique EDL quantifier and it is referred as the isoelectric point (IEP). In Fig. 1b we show the measured pH-dependencies of the $u_{e}$ and $\zeta$-potential for the hematite particles at different $\mathrm{pH}$ values. The presented $u_{e}$ and $\zeta$ values are the maximalintensity ones that are averaged over several measurements. The observed IEP (6.1) is consistent with the theoretically predicted point of zero charge (PZC) of the (001) crystal face of hematite [25, 26, 27].

Calculating $\zeta$-potential from $u_{e}$. As shown in Fig. 2b the hematite aggregate diameter is between 25 and $650 \mathrm{~nm}$ in the considered pH-range, this corresponds to $\kappa a$ values between 120 and 3126 - clearly in the thin-double layer regime ( $\kappa a \gg 1$, Smoluchowski eq. (2)). In addition, the values of $|\zeta|$ are below $50 \mathrm{mV}$ (Fig. 1b), and $u_{e}$ decreases with increasing $\mathrm{KNO}_{3}$ concentration confirming that a stagnant-layer conductivity and concentration polarization are negligible.

In Fig. 2a,b we present the probability distribution functions of the electrophoretic mobility $\left(u_{e}\right.$, Fig. $\left.2 \mathrm{a}\right)$ and the hydrodynamic diameter $\left(d_{\text {hydro }}\right.$, Fig. $\left.2 b\right)$. The distributions are obtained by averaging individual histograms collected in seven independent measurements, and fitting to a Gaussian function (see Supporting Information).

As the $\mathrm{pH}$ approaches the IEP the absolute values of the electrophoretic mobility and $\zeta$ potential decrease (Figs. 1b, 2a). As a result the electrostatic repulsion - essential in suspension stability - weakens and particles start to aggregate (see change in $d_{\text {hydro }}$, Fig. 2b). The structure and density of aggregates are critical for their potential influence to overprint the size-dependent reactivity (e.g., reductive dissolution $[14,28]$ ), so our proposed method is potentially useful for relating the electrokinetic potential to those physical characteristics of particle aggregates.

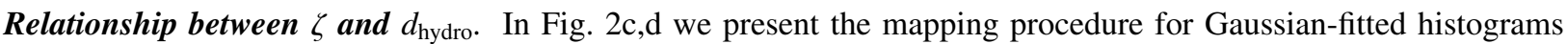
of $u_{e}$ and $d_{\text {hydro }}$. In order to carry out the mapping we divided the function domain into two reversible subdomains (see Supporting Information). Note that our mapping procedure requires prior knowledge of whether $|\zeta|$ increases or decreases with aggregate size. In principle, aggregates should obey the same relationship between $u_{e}$ and size as observed for monodispersed suspensions with varying particle sizes.

Unfortunately, reported experimental data provide an unclear relationship between particle size and $\zeta$ potential. For instance, He et al. [29] and Sheng et al. [30] showed that $|\zeta|$ increases with increasing particle size, whereas Madden et al.[31] reported an opposite trend (i.e., $|\zeta|$ decreases with increasing particle size).

He et al. [29] showed that as the particle size decreases the aggregation rate increases and the critical coagulation concentration decreases. This suggests that smaller particles are less charged than the larger ones and their electrostatic repulsion is screened at lower concentration of background electrolyte. However, as the ionic strength increases the size-potential relationship is less pronounced due to thinner electrical double layers around particles and more screened electrostatic repulsive forces[29, 30]. For instance, He et al.[29] and Sheng et al. [30] reported that there is no significant difference in $\zeta$ potential between 12 and $65 \mathrm{~nm}$ particles (for $100 \mathrm{mM} \mathrm{NaCl}$ )[29] and between 12 and $65 \mathrm{~nm}$ particles (for $60 \mathrm{mM} \mathrm{NaCl}$ )[30]. What is more, Madden et al. [31] have reported that $|\zeta|$ is larger for $7 \mathrm{~nm}$ particles than for $25 \mathrm{~nm}$ particles (for $100 \mathrm{mM} \mathrm{NaCl}$ ).

In the case of dilute electrolyte solution, $|\zeta|$ should decrease with particle size[29, 30], therefore we map $P\left(d_{\text {hydro }}\right)$ onto $P\left(-u_{e}\right)$ for $\mathrm{pH}>\mathrm{IEP}$ and $P\left(d_{\text {hydro }}\right)$ onto $P\left(u_{e}\right)$ for $\mathrm{pH}<\mathrm{IEP}$ (Fig. $\left.2 \mathrm{c}, \mathrm{d}\right)$. If on the other hand $|\zeta|$ increases with particle size, as observed by Madden et al. [31], one should reverse the mapping (i.e., $P\left(d_{\text {hydro }}\right) \rightarrow P\left(-u_{e}\right)$ for $\mathrm{pH}<\mathrm{IEP}$, $P\left(d_{\text {hydro }}\right) \rightarrow P\left(u_{e}\right)$ for $\left.\mathrm{pH}>\mathrm{IEP}\right)$.

Mapping of aggregate diameter onto $\zeta$-potential is equivalent in our case as the Smoluchowski relation is valid for our conditions (see Supporting Information).

In Fig. 3 we show the size-dependencies of the electrophoretic mobilities and corresponding $\zeta$-potential obtained by applying the distribution mapping procedure. Surprisingly, both $u_{e}$ and corresponding $\zeta$-potential are linear functions of the particle (aggregate) size. Although Smoluchowski's formalism is valid for our system, we have also presented in Fig. 3 the $\zeta$-potential values obtained using Hückel (eq. (3)) and Henry's formulas (eq. (4)). 
Size-dependent particle acidity. To understand why the values of $\left|u_{e}\right|$ and $|\zeta|$ increases with increasing particle size, we generated a number of hypothetical spherical $\alpha-\mathrm{Fe}_{2} \mathrm{O}_{3}$ particles and analyzed the populations of surface protonreactive groups $\left(\equiv \mathrm{Fe}_{n} \mathrm{O}\right.$, where $n$ indicates Fe-coordination of the surface $\mathrm{O}$ atoms). Our modeling is based on the crystal structure reported by Blake et al. [32]. Not surprisingly, this analysis shows that as the particle size increases, the molecular-scale curvature decreases, which leads to more complete coordination of surface exposed oxygen atoms to lattice $\mathrm{Fe}$ (triply coordinated $\equiv \mathrm{Fe}_{3} \mathrm{O}$ in particular, see Supporting Information). Because these triply coordinated surface sites are less reactive [25, 26], the surface becomes less acidic, consistent with observed increases of the IEP and PZC values with increasing particle size. This less acidic surface is also more inert in terms of surface reactivity[31, 33, 34, 35, 36, 37, 38].

In summary, we calculated that the particle-size dependence of PZC and IEP values originates from the decrease of surface acidity due to an increasing proportion of higher-coordinated and less acidic surface sites with increasing particle size (see Supporting Information).

However, particle-size-dependent reactivity is also affected by other factors including particle morphology, porosity, structure (crystal vs aggregate), and type of surface reactions considered [14, 39, 40]. For instance, Lanzl and coworkers $[14,28]$ reported that the aggregate size has little effect on the rate of reductive dissolution, which in contrast is affected by the size of primary particles (aggregate grains) [14, 28].

It is important to emphasize that the linear relationships $u_{e}=f\left(d_{\text {hydro }}\right), \zeta=f\left(d_{\text {hydro }}\right)$ are valid only within the experimental domains of $u_{e}$ and $d_{\text {hydro. }}$. What is more, experimental histograms of $d_{\text {hydro }}$ and $u_{e}$ may not provide a monotonic distribution function, if this is the case then our procedure provides an unambiguous mapping only for the monotonic subdomains of the distributions. Although the mapping procedure applied for hematite particles assumes that $|\zeta|$ increases with increasing particle size as suggested by He et al. [29] and Sheng et al. [30], the observed linearity is independent of that assumption.

\section{Conclusions}

In this report, we propose a mapping of the histogram of hydrodynamic diameter onto the histogram of electrokinetic potential to provide insight into the size-dependencies of the electrophoretic mobility $\left(u_{e}\right)$ and corresponding $\zeta$-potential for a model iron oxide - hematite $\left(\alpha\right.$-Fe $\left.\mathrm{O}_{3}\right)$. It is generally accepted that reactivity of hematite nanoparticles decreases with increasing particle size, however the underlying cause of that phenomenon has not, until now, been sufficiently explained [14].

Here, we found that that $u_{e}$ and $\zeta$-potential increase linearly with increasing particle size. This is a manifestation of size-dependent electrochemical reactivity of hematite particles, which is caused by an increasing proportion of inert, triply coordinated surface groups $\left(\equiv \mathrm{Fe}_{3} \mathrm{O}\right)$ as the particle size increases. A change in the population density of the most reactive surface group as the particle size changes explains why smaller particles are more acidic than larger ones.

Furthermore, our procedure allows one to fully utilize the data collected by commercial ZPAs, which otherwise are often disregarded. We believe that the probability mapping can have a much broader application that we demonstrated here for $\alpha-\mathrm{Fe}_{2} \mathrm{O}_{3}$ particles. Such mapping strategies can be useful in studying a size-dependent surface electrostatics of biologically relevant particles, including viruses and bacteria.

\section{Acknowledgements}

This work was supported by the Geosciences Research Program at PNNL sponsored by the U.S. Department of Energy (DOE), Office of Science, Office of Basic Energy Sciences (BES), Division of Chemical Sciences, Geosciences $\&$ Biosciences. A portion of this research was performed using EMSL, a national scientific user facility sponsored by the DOE's Office of Biological and Environmental Research and located at Pacific Northwest National Laboratory. Pacific Northwest National Laboratory (PNNL) is a multiprogram national laboratory operated for DOE by Battelle. K.K.K. was supported by the Ministry of Science and Higher Education (grant IP2012 059872). 

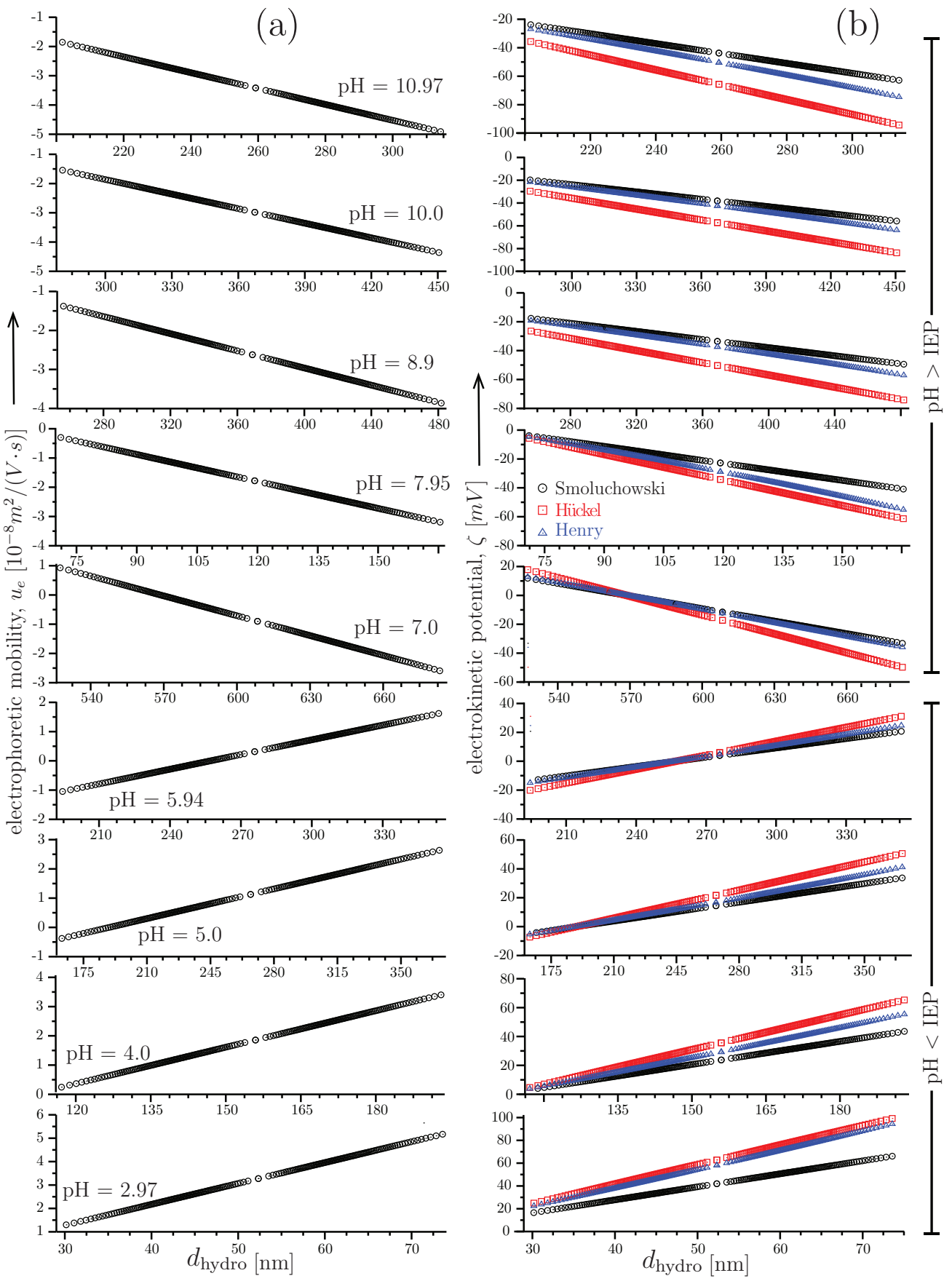

Figure 3: Size-dependencies of $u_{e}$ and $\zeta$-potential of the hematite particles at various $\mathrm{pH}$ - obtained by mapping $P\left(u_{e}\right)$ and $\left.P\left(d_{\mathrm{hydro}}\right)\right)$ distributions. The mapped mobility (left-hand side, $u_{e}=f\left(d_{\text {hydro }}\right)$ ) is converted to $\zeta$-potential (right-hand side) using Smoluchowski (eq. (2)), Hückel (eq. (3)) and Henry/Ohshima's (eqs. $(4,5)$ ) formalisms. See Supporting Information for mapping details.

\section{References}

[1] J. Lyklema, Fundamentals of Interface and Colloid Science, Vol. 2, Academic Press, San Diego, 1995.

[2] D. J. Shaw, Introduction to Colloid and Surface Chemistry, 4th Edition, Butterworth Heinemann, Oxford, 1992.

[3] S. E. Harding, Determination of Diffusion Coefficients of Biological Macromolecules by Dynamic Light Scattering, Methods in Molecular Biology 22 (1994) 97-108. 
[4] B. J. Berne, R. Pecora, Laser Light Scattering from Liquids, Annu. Rev. Phys. Chem. 25 (1974) 233-253.

[5] V. A. Bloomfield, Quasi-Elastic Light Scattering Applications in Biochemistry and Biology, Annu. Rev. Biophys. Bioeng. 10 (1981) $421-450$.

[6] J. M. Schurr, K. S. Schmitz, Dynamic Light Scattering Studies of Biopolymers: Effects of Charge, Shape, and Flexibility, Annu. Rev. Phys. Chem. 37 (1986) 271-305.

[7] W. W. Wilson, M. M. Wade, S. C. Holman, F. R. Champlin, Status of Methods for Assessing Bacterial Cell Surface Charge Properties Based on Zeta Potential Measurements, J. Microbiol. Methods 43 (2001) 153-164.

[8] Y. Zhang, M. Yang, N. G. Portney, D. Cui, G. Budak, E. Ozbay, M. Ozkan, C. S. Ozkan, Zeta Potential: a Surface Electrical Characteristic to Probe the Interaction of Nanoparticles with Normal and Cancer Human Breast Epithelial Cells, Biomed. Microdevices 10 (2007) 321-328.

[9] A. V. Delgado, Interfacial Electrokinetics and Electrophoresis, Vol. 106 of Surfactant Science Series, Marcel Dekker, Inc., New York, 2002.

[10] H. G. Barth, R. B. Flippen, Particle Size Analysis, Anal. Chem. 67 (1995) 257R-272R.

[11] B. B. Weiner, W. W. Tscharnuter, Uses and Abuses of Photon Correlation Spectroscopy in Particle Sizing, in: Provder, T (Ed.), Particle Size Distribution. Assessment and Characterization, 1987, Ch. 3.

[12] E. Gulari, A. Annapragad, E. Gulari, B. Jawad, Determination of Particle Size Distributions Using Light-Scattering Techniques, in: Provder, T (Ed.), Particle Size Distribution. Assessment and Characterization, 1987, Ch. 9.

[13] J. J. Carlson, S. K. Kawatra, Factors Affecting Zeta Potential of Iron Oxides, Miner. Process. Extr. Metall. Rev. 34 (2013) 269-303.

[14] C. A. Lanzl, Size-dependent reactivity of hematite nanoparticles: Environmental implications of dissolution mechanisms and aggregation, Ph.D. thesis, University of Iowa, Iowa (May 2013).

[15] B. J. Berne, P. R, Dynamic Light Scattering with Application to Chemistry, Biology, and Physics, Dover, Mineola, 2000.

[16] D. Sidebottom, Dynamic Light Scattering, in: Kaufmann, E N (Ed.), Characterization of Materials, 2nd Edition, Wiley-Blackwell, 2012.

[17] A. V. Delgado, F. González-Caballero, R. J. Hunter, L. K. Koopal, J. Lyklema, Measurement and Interpretation of Electrokinetic Phenomena, J. Colloid Interface Sci. 309 (2007) 194-224.

[18] H. Ohshima, A Simple Expression for Henry's Function for the Retardation Effect in Electrophoressis of Spherical Colloidal Particles, J. Colloid Interface Sci. 168 (1994) 269-271.

[19] H. Ohshima, Electrophoresis, in: M. Akay (Ed.), Encyclopedia of Biomedical Engineering, Wiley, Hoboken, 2006, pp. 1443-1449.

[20] inverse of function $f: X \rightarrow y$ defined as $y=f(x)$ is given by $f^{-1}: Y \rightarrow x$ defined by $x=f^{-1}(y)$.

[21] W. Schärtl, Light Scattering from Polymer Solutions and Nanoparticle Dispersions, Springer, Berlin, 2007.

[22] R. Kubo, M. Toda, N. Hashitsume, Statistical Physics II. Nonequilibrium Statistical Mechanics, Springer-Verlag, Berlin, 1985.

[23] K. L. Ngai, Relaxation and Diffusion in Complex Systems, Springer, New York, 2011.

[24] M. Kosmulski, Surface Charging and Points of Zero Charge, CRC Press, Boca Raton, 2009.

[25] T. Hiemstra, P. Venema, W. H. V. Riemsdijk, Intrinsic Proton Affinity of Reactive Surface Groups of Metal (Hydr)oxides: The Bond Valence Principle, J. Colloid Interface Sci. 184 (1996) 680-692.

[26] P. Venema, T. Hiemstra, P. G. Weidler, W. H. van Riemsdijk, Intrinsic Proton Affinity of Reactive Surface Groups of Metal (Hydr)oxides: Application to Iron (Hydr)oxides, J. Colloid Interface Sci. 198 (1998) 282-295.

[27] P. Zarzycki, S. Chatman, T. Preočanin, K. M. Rosso, Electrostatic Potential of Specific Mineral Faces, Langmuir 27 (2011) 7986-7990.

[28] C. A. Lanzl, J. Baltrusaitis, D. M. Cwiertny, Dissolution of Hematite Nanoparticle Aggregates: Influence of Primary Particle Size, Dissolution Mechanism, and Solution pH, Langmuir 28 (2012) 15797-15808.

[29] Y. T. He, J. Wan, T. Tokunaga, Kinetic Stability of Hematite Nanoparticles: the Effect of Particle Sizes, J. Nanopart. Res. 10 (2007) $321-332$.

[30] A. Sheng, F. Liu, L. Shi, J. Liu, Aggregation Kinetics of Hematite Particles in the Presence of Outer Membrane Cytochrome OmcA of Shewanella oneidenesis MR-1, Environ. Sci. Technol. 50 (2016) 11016-11024.

[31] A. S. Madden, M. F. Hochella, T. P. Luxton, Insights for Size-Dependent Reactivity of Hematite Nanomineral Surfaces through $\mathrm{Cu}^{2+} \mathrm{Sorption}$ Geochim. Cosmochim. Acta 70 (2006) 4095-4104.

[32] R. L. Blake, R. E. Hessevick, T. Zoltai, L. W. Finger, Refinement of the Hematite Structure, Am. Mineral. 51 (1966) 123-129.

[33] A. J. Anschutz, R. L. Penn, Reduction of Crystalline Iron(III) Oxyhydroxides Using Hydroquinone: Influence of Phase and Particle Size, Geochem. Trans. 6 (2005) 60-7.

[34] Z. Abbas, C. Labbez, S. Nordholm, E. Ahlberg, Size-Dependent Surface Charging of Nanoparticles, J. Phys. Chem. C 112 (2008) 5715-5723.

[35] G. Rubasinghege, R. W. Lentz, M. M. Scherer, V. H. Grassian, Simulated Atmospheric Processing of Iron Oxyhydroxide Minerals at Low pH: Roles of Particle Size and Acid Anion in Iron Dissolution, Proc. Natl. Acad. Sci. U.S.A. 107 (2010) 6628-6633.

[36] G. Rubasinghege, R. W. Lentz, H. Park, M. M. Scherer, V. H. Grassian, Nanorod Dissolution Quenched in the Aggregated State, Langmuir $26(2010)$ 1524-1527.

[37] G. Rubasinghege, P. K. Kyei, M. M. Scherer, V. H. Grassian, Proton-Promoted Dissolution of $\alpha$-FeOOH Nanorods and Microrods: Size Dependence, Anion Effects (Carbonate and Phosphate), Aggregation and Surface Adsorption, J. Colloid Interface Sci. 385 (2012) 15-23.

[38] D. M. Cwiertny, G. J. Hunter, J. M. Pettibone, M. M. Scherer, V. H. Grassian, Surface Chemistry and Dissolution of $\alpha$-FeOOH Nanorods and Microrods: Environmental Implications of Size-Dependent Interactions with Oxalate †, J. Phys. Chem. C 113 (2009) 2175-2186.

[39] B. Gilbert, R. K. Ono, K. A. Ching, C. S. Kim, The Effects of Nanoparticle Aggregation Processes on Aggregate Structure and Metal Uptake, J. Colloid Interface Sci 339 (2009) 285-295.

[40] T. Echigo, D. M. Aruguete, M. Murayama, M. F. Hochella, Influence of Size, Morphology, Surface Structure, and Aggregation State on Reductive Dissolution of Hematite Nanoparticles with Ascorbic Acid, Geochim. Cosmochim. Acta 90 (2012) 149-162. 


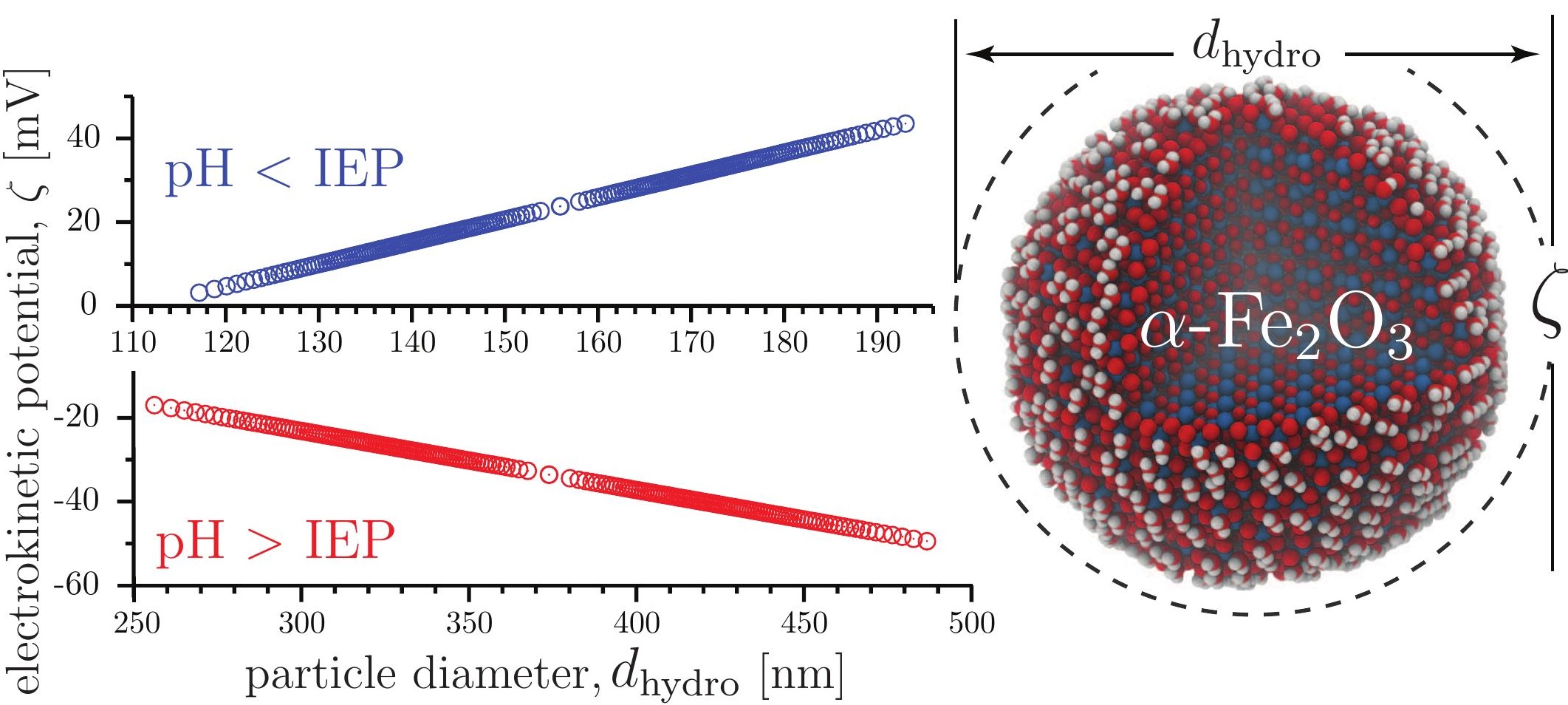

